\title{
Turismo y Cultura: Reflexión de su Práctica en Afro Comunidades de Latinoamérica y el Caribe
}

\section{Tourism and culture: reflection about its practice in afro communities of Latin America and the Caribbean}

\begin{abstract}
RESUMEN
La discusión sobre el uso del patrimonio cultural en el ámbito turístico es constante e interminable ¿Cómo la cultura se muestra a otros con fines socioeconómicos, comerciales o políticos? ¿Es el turismo un factor de escenarios no favorables para el bienestar de las comunidades? Si el patrimonio cultural es lo que da sentido de pertenencia y la manifestación de la persona en su entorno social con el fin de generar su propio bienestar. Entonces la decisión de elegir un medio le corresponde a sí mismo. Sin embargo, la desigualdad y la inequidad son brechas que no permiten el bien común. Palabras claves: Turismo; patrimonio; cultura afro; igualdad; equidad.
\end{abstract}

\section{ABSTRACT}

The discussion about the use of the cultural heritage in the tourist context in permanent and unfinished. ¡How the culture is show to others with socioeconomic, commercial or political goals? ¿Is the tourism a factor of non beneficial situation for the communities' wellness? If the cultural heritage is what give sense of integration and the expression of the persons in their social environment with the purpose of generate their own wellness. So, the decision of choose a way correspond to themselves. Nevertheless, the inequality and the inequity are gaps that do not allow the common benefit.

Keywords: Tourism; heritage; afro culture; equality; equity.

\section{Samanta Hilda Calle Ruiz}

samanta.calle@gmail.com

Universidad Nacional Mayor de San Marcos, Facultad de Ciencias Administrativas. Lima, Perú 


\section{INTRODUCCIÓN}

La relación entre la cultura y el turismo ha sido el eje de diversos debates respecto al uso del patrimonio para la generación del desarrollo local sostenible en beneficio de la comunidad. Partiendo del enfoque antropológico, la discusión en torno al turismo ha estado relacionada con la autenticidad de la cultura, la identidad, los beneficios otorgados para el desarrollo humano y los impactos que genera (Fuller, 2009).

Es decir, la cultura como performance momentáneo con fines turísticos que conlleva a la pérdida de identidad, consolidación o transformación de la misma; la generación de beneficios para los locales o los agentes externos; el logro de un cambio estructural con respecto a los problemas que aqueja a la sociedad como la pobreza, la violencia, la desnutrición, entre otros; los impactos positivos o negativos en el entorno, sea medio ambiental, social o cultural. En pocas palabras, el cuestionamiento si esta relación cultura y turismo trae bienestar para algunos o trae el bien común.

Actualmente, las diversas manifestaciones del hombre, los aportes a la memoria histórica y la identidad han logrado constituir grupos sociales diversos que, en Latinoamérica, en muchos casos, son excluidos e invisibilizados aún. Pero el reconocimiento de los aportes culturales de los ciudadanos es esencial para construir identidad y lograr la participación en acciones de desarrollo sostenible. La invisibilización de la cultura afrodescendiente es uno de los casos evidentes en la sociedad, pues siendo una de las más representativas como fenómeno social, es la que más demandas no atendidas tiene.

Para entender la significancia de las acciones en turismo en pro del bienestar del ser humano se inicia por recalcar lo que el Código Ético Mundial para el Turismo de la Organización Mundial del Turismo (OMT, 1999), en su artículo 2 inciso 2, indica: el quehacer turístico respetara la igualdad entre mujeres y hombre. Igualmente se encauzará a promover los derechos humanos, en especial de grupos de población vulnerable conformada por la infancia, la tercera edad, las minorías étnicas y pueblos originarios. Como se evidencia, el enunciado demanda la igualdad y atención a los más desfavorecidos, considerándose esto último como equidad. Ya que la atención prioritaria a los derechos de las poblaciones vulnerables significa colocarlos en la esfera de la igualdad para atender sus demandas.

\section{OBJETIVO DEL ENSAYO}

Reflexionar sobre el aporte significativo y/o beneficio de la actividad turística a los propósitos socioculturales de los afrodescendientes en Latinoamérica y el Caribe.

\section{ARGUMENTACIÓN}

Para brindar un punto de vista particular con respecto a la práctica del turismo y la cultura en una relación primero se recurre a reflexionar sobre las definiciones conceptuales de turismo y cultura, luego las demandas de los afrodescendientes y finalmente las conclusiones de diversos estudios que analizan estas prácticas en afro comunidades.

\section{Turismo y cultura.}

La definición consensuada del turismo y afirmada por la OMT (s.f) es la siguiente: fenómeno económico social y cultural afín con el traslado de personas a sitios fuera de su entorno habitual de residencia, sea por motivaciones profesionales o personales. Denominando a estas personas como visitantes, y el turismo tiene que ver con sus actividades, que envuelven un gasto turístico.

Nótese el reconocimiento del turismo como una manifestación social y cultural antes que económica. Recuérdese la economía como una manifestación cultural del ser humano. Por tanto, el turismo es una dinámica sociocultural esencialmente. En esta sociedad contemporánea en la que prima la globalización, el turismo es una manifestación consecuente de ella. También se hace hincapié a las motivaciones, es decir, a la razón que impulsa a trasladarse a una persona a otro escenario geográfico- social en donde es parte de una dinámica que se da a partir de su llegada.

Con respecto a la cultura la Organización de Las Naciones Unidas (UNESCO) declara desde 1982 que la cultura es el acervo de atributos materiales, intelectuales, afectivos y espirituales que distinguen a un grupo social. Ello incluye del mismo modo que la literatura y el arte, el 
modo de vida, los derechos del ser humano, los modos de vida, los derechos del ser humano, los valores, las tradiciones y las creencias. Admite la reflexión de la persona sobre sí misma. Es la que hace humanos racionales, críticos y éticamente comprometidos. A través de esta se establecen valores que guían las acciones. La persona se expresa, toma conciencia sobre sí misma, se reconoce como un proyecto inconcluso, discute sus relaciones, e indaga nuevos significados y crea obras que le habilita para trascender.

Si nos centramos en la última parte de esta definición de cultura, se puede decir que se reconoce al ser humano como aquel que está en una búsqueda constante de sentido llevándolo a crear nuevas obras para trascender.

Entonces en esta búsqueda del trascender del ser humano ¿no es el acto de mostrarse un medio?, ¿cómo trasciende el ser humano si es invisibilizado? ¿Podría decirse que la recreación de sus obras para mostrarse al turismo es posiblemente el medio? Sin embargo, también refiere a los derechos, la reflexión del ser humano sobre sí mismo y al compromiso ético que tiene en base a la cultura. ¿Entonces en la actividad turística se considera los derechos el sentido y la ética?

\section{Demandas de los afrodescendientes.}

En América Latina la población afrodescendiente está conformada por personas descendientes de africanos esclavizados durante la trata de personas que duro casi 400 años. De acuerdo a la Comisión Económica para América Latina y el Caribe (CEPAL):

Conocer la cantidad de personas afrodescendientes en América Latina continúa siendo uno de los desafíos más básicos y urgentes, puesto que resulta difícil brindar una cifra acertada debido a los problemas relacionados con la identificación étnico-racial en las fuentes de datos, que van desde la falta de inclusión de preguntas pertinentes hasta la calidad de la información recogida (CEPAL, 2017, p. 51).

Ante esta situación, CEPAL realizo una estimación a partir del último censo y estimaciones al 2010 de 16 países de Latinoamérica y el Caribe. En la Tabla 1 se detalla resultados del porcentaje de población afrodescendiente respecto del total poblacional.

De acuerdo con la resolución ratificada por la Asamblea de las Naciones Unidas el 18

Tabla 1

América Latina (16 países): población afrodescendiente según último censo y estimaciones a 2010

\begin{tabular}{|c|c|c|c|}
\hline Países & $\begin{array}{c}\text { Población } \\
\text { total }\end{array}$ & $\begin{array}{c}\text { Población afrodescen- } \\
\text { diente total }\end{array}$ & $\begin{array}{c}\text { Porcentaje afrodes- } \\
\text { cendiente }\end{array}$ \\
\hline \multicolumn{4}{|c|}{ Resultados y fechas censales } \\
\hline Argentina 2010 & 40117096 & & 0,4 \\
\hline Bolivia (estado plurinacional de) 2012 & 10059856 & & 0,2 \\
\hline Brasil 2010 & 190755799 & & 50,9 \\
\hline Costa Rica 2011 & 4301712 & & 7,8 \\
\hline Cuba 2012 & 11167325 & & 35,9 \\
\hline Ecuador 2010 & 14483499 & & 7,2 \\
\hline Honduras 2013 & 8303772 & & 1,4 \\
\hline México 2010 & 112336538 & & 1,2 \\
\hline Panamá 2010 & 3405813 & & 8,8 \\
\hline Uruguay 2011 & 3251654 & & 4,6 \\
\hline Venezuela (República Bolivariana de) 2011 & 27227930 & & 3,4 \\
\hline \multicolumn{4}{|l|}{ Estimaciones a 2010} \\
\hline Colombia & 46448000 & & 10,5 \\
\hline El salvador & 6218000 & & 0,1 \\
\hline Guatemala & 14334000 & & 0,04 \\
\hline Nicaragua & 5813000 & & 0,5 \\
\hline Perú & 29272000 & & 2,0 \\
\hline Total & 527495994 & & 21,1 \\
\hline
\end{tabular}

Fuente: Comisión Económica para América Latina y el Caribe (CEPAL), 2017 
de noviembre del 2014 de las Naciones Unidas "El Decenio Internacional de los Afrodescendientes: Reconocimiento, Justicia y Desarrollo 2015 - 2024" debe propiciar el respeto, amparo y práctica de los derechos humanos y libertad esenciales de los afrodescendientes, como se contempla en la Declaración Universal de Derechos Humanos".

Entonces el propósito, en este periodo en que se vive todas las manifestaciones, movimientos, discursos, demandas a nivel internacional por parte de activistas, líderes, instituciones públicas, organizaciones, entre otros, como representantes de los afrodescendientes, es garantizar el ejercicio de sus derechos y libertad que han sido negados en diversas medidas y formas a consecuencia de siglos de esclavitud.

Becerra (2008) menciona que las estrategias de visibilización afrodescendiente en el nuevo mileno se centran en la visibilización estadística y la organización, con respecto a la primera aún hay dificultades por no tener datos estandarizados, sino más bien diversos criterios para obtener datos que reflejen cuantos son y cuál es la situación de los afrodescendientes. Hoy los niveles de organización a nivel internacional están permitiendo escuchar la voz de movimientos en favor de las políticas culturales y sociales de afrodescendientes con diversos representantes a nivel de Latinoamérica y el Caribe.
Así también, se tiene como expectativa que el decenio aporte a una considerable visibilidad de los afrodescendientes, no solo estadísticamente sobre el ejercicio de sus derechos a la auto identificación en censos, encuestas y registros administrativos, sino además a la implementación y fortalecimiento de marcos institucionales que soporten la coordinación de políticas de inclusión dirigidas al vencimiento del racismo y de las desigualdades étnico-raciales (Comisión Económica para América Latina y el Caribe, 2017).

Se comprende que el ejercicio de libertades y derechos de los afrodescendientes se lograran en la medida que se reduzcan o corten las brechas de desigualdad a causa de la discriminación, racismo y circunstancias que los colocaron en situaciones de inequidad social. En la Figura 1 se observa que la vulnerabilidad a la pobreza de personas afrodescendientes respecto de los no afrodescendientes es mayor en los cuatro países objeto del análisis. Esto refleja que la desventaja es mayor en los descendientes de africanos esclavizados pese a ser una minoría.

Si se expone y cuestiona al turismo como medio para estos propósitos es por dos razones: la primera porque con respecto al desarrollo la Organización de los Estados Americanos en su plan de acción del Decenio de los y las Afrodescendientes en las Américas (2016 - 2025, p. 12)

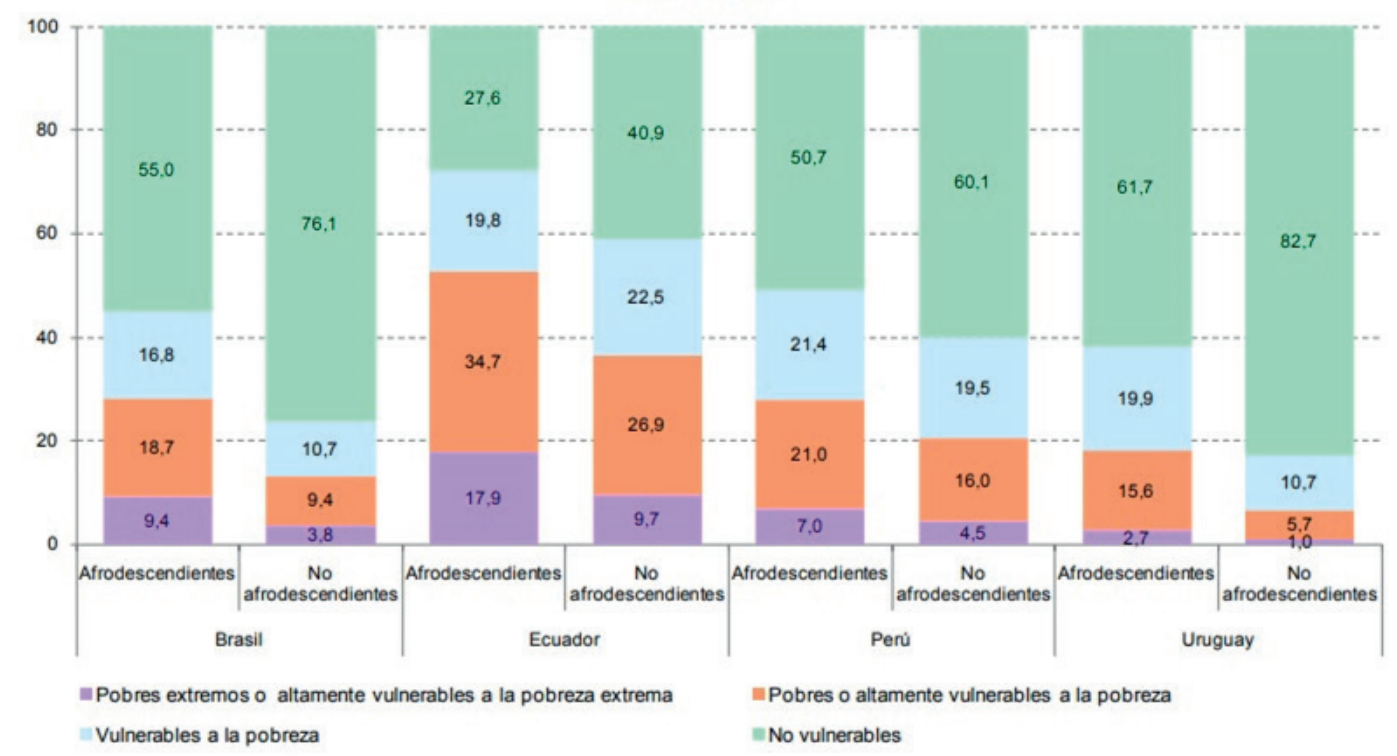

Figura 1. América Latina (4 países): Vulnerabilidad a la pobreza de la población total clasificado en afrodescendientes y No afrodescendientes. CEPAL (2017). 
plantean dos objetivos relacionados con el turismo, siendo estos:

- Promover la inclusión de la población afrodescendiente en los programas de ciencia, innovación, y tecnología, comercio y empoderamiento económico, cultura y turismo, desarrollo sostenible y medio ambiente, en particular en comunidades con presencia afrodescendiente.

- Fomentar la inclusión de industrias culturales y turísticas en comunidades con presencia afrodescendiente.

La segunda razón es porque en muchas comunidades de presencia afrodescendiente se está promoviendo la industria turística cultural. Pero, ¿el turismo representa para esas poblaciones el medio para generar logros significativos en el camino a su visión de futuro como comunidad internacional? A continuación, se hace referencia a diversas posturas en el análisis de experiencias en la región.

\section{El sentido del turismo en comunidades de cultura afrodescendiente.}

La experiencia en Panamá ha sido favorable para los locales de las Antillas, según Guerron (2003) la actividad turística facilita el avance de una política cultural compleja, para mejorar su sitio en el multiculturalismo panameño $y$, a su vez, erige identidades nacionales y diaspóricas.

Es decir, ahora los antillanos del archipiélago de Bocas de Toro participan del desarrollo turístico del país, construyen identidades y medios que les permite aspirar a un cambio en su nivel de vida. Ellos aspiran a la tecnología, la infraestructura y oportunidades de bienes y servicios públicos. Aunque también empiezan a formar parte de la nación, se desarrolla una estructura de identidades para preservar aquello que sostiene el turismo que es la singularidad, autenticidad, lo diferente que busca el turista en el otro. En otras palabras, el turismo permite la conservación de diversas identidades de los afroantillanos y a la vez la participación en el desarrollo de la nación panameña. No se niega tampoco las consecuencias en el patrimonio natural y el sacrifico de este para atender a la demanda.
Por otro lado, Buitrago (2006), en su análisis del desarrollo turístico en Boquilla, Cartagena, afirma que dicho desarrollo ha perjudicado a las poblaciones afrodescendientes que han ocupado la zona por muchos años. El desarrollo sostenible que se divulga es con fines de conservación de los ecosistemas para beneficio del desarrollo turístico de playas. Pero no se menciona los impactos sociales que ocasiona en una población que se instaló previamente en la zona y que está siendo excluida y obligada a salir del territorio ocupado, esto con fines de instalación de infraestructura turística para el desarrollo de turismo basándose en la oferta de servicios hoteleros para un turismo de masas y de exigencia de servicios de lujo que obviamente la población local no puede ofertar, generando la perdida de sus costumbres y la desestructuración de su comunidad como construcción social que se ha dado.

Las consecuencias no favorables también se evidencian por Campos y Moquete (2008), en el estudio realizado para analizar el movimiento afro en Ilhéus, Bahía, Brasil en el contexto de las industrias creativas como modelo de desarrollo turístico local, indican que en este contexto de inequidades socioeconómicas y una realidad actual neoliberal se restringen la defensa de cambios de políticas sociales por las cuales nacen los movimientos sociales afro. No existe el poder local para la gestión de su propio desarrollo, no se consideran el mercado externo como interno, no hay inversión del estado, persiste la exclusión de grupos afro en las políticas sociales del estado y el mercado interno es no significativo. Se usa a los grupos culturales como instrumento de desarrollo de políticas del turismo y no como grupos con vocación cultural y política. Actualmente los movimientos de grupos afro en el lugar tienen una perspectiva de identidad y diferencia para su desenvolvimiento. Sin embargo, negocian peligrosamente con la lógica empresarial. Entonces la situación es enfrentar los propósitos externos a los propósitos por los cuales se originaron, para no desviarse del camino de la visibilización político cultural, para lograr incluirse en el bienestar que se supone el desarrollo socioeconómico brinda.

Para reflexionar sobre el sentido del turismo para la gestión de la cultura, se puede repen- 
sar lo que Pérez Amores (2010) menciona que se han unido tres esferas que convivían en este ambiente social sin que previamente hubiesen conectado verdaderamente: religión-patrimonio-turismo, un triángulo en que la economía es el foco. Patrimonio y turismo ensamblados bajo la cara de la religión enlazados con una ideología y una identidad, en donde la poderosa presencia de la fe y la tradición, pero además de la mercancía, el debate político y la globalización muestran diferentes caras de la construcción del patrimonio cultural.

Hoy en día, se debe considerar detenidamente si las prácticas para la construcción del patrimonio cultural, a partir de un enfoque económico, en este caso uniendo las concepciones de patrimonio y turismo con una manifestación cultural (para preservarla o modificarla), aporta realmente a promover la cultura como eje de desarrollo y no solo como recurso para la instauración de productos turísticos culturales. Esto con fines de rentabilidad y sostenibilidad económica en el tiempo. ¿Cuál es el rol de la cultura es una pregunta a hacerse cuando vamos a hacerla interactuar con la industria turística?

A continuación, se adicionan las reflexiones sobre otros estudios que, al analizarlos, sugieren como respuesta a esta interrogante considerar a la cultura como pilar para la gestión del desarrollo de las comunidades. Donde se valora al turismo como una oportunidad para visibilizar. Pero, atendiendo al hecho que, si el centro de atención son las manifestaciones culturales como recursos turísticos para tener un show que mostrar, indudablemente se perderá la potencia que representaría el turismo como puente de comunicación y acción para el logro de la reivindicación, la justicia y el desarrollo que profesa el decenio de los afrodescendientes. La cultura de acuerdo a la UNESCO no solo se refiere a manifestaciones materiales e inmateriales de expresión artística, también son manifestaciones de la ética, los derechos, la ciencia, la política, entre otras diversas formas que permiten la interacción entre seres humanos para el logro de su bienestar a partir del logro del consenso. Para decirlo de otra manera, el bien común a pesar de las diferencias culturales.

Por ejemplo, Tresserras (2013) indica que el reto para el desarrollo del turismo de base comunitaria es alcanzar estrategias de mercadeo óptimas, bajo una gestión endógena, revalorando la cultura y generando empoderamiento de las comunidades. Esto quiere decir que no se debe perder el horizonte con respecto a que el desarrollo sostenible toma en cuenta la cultura como pilar fundamental. Sin embargo, mayormente se usa como mero recurso.

Camargo y Lawo-Sukam (2015) en su estudio concluyen que, en San Basilio de Palenque, la comunidad ha sobrevivido con su cultura gracias a su organización social y practica de sus manifestaciones. Sin embargo, sugieren que el turismo étnico puede consolidar el desarrollo económico para su buen vivir y la sostenibilidad cultural, siempre y cuando no sea un turismo no planificado. Se debe considerar principios de igualdad y justicia para la distribución de beneficios y toma de decisiones. Ellos afirman que es necesaria la participación no solo de los palenqueros, sino además del estado y la industria turística para preservar y proteger la herencia afro cultural de la comunidad de San Basilio.

Benítez y Albuja (2015) sugieren la revalorización de personajes afro a través del turismo. Sin embargo, sugieren también que el estado debe promover las redes de turismo y políticas del buen vivir para dar soporte a proyectos culturales, considerando al turismo como una línea estratégica para la visibilización, revalorización y generación de oportunidades de desarrollo.

Pazos (2016) indica que, en diversos escenarios de la ciudad de Cali, Colombia el estado nación promueve las industrias culturales para el turismo, centrándose en las expectativas y demandas de los turistas y/o visitantes. El discurso propone la valoración de la cultura. Sin embargo, los propios actores, protagonistas de la cultura afro pacífica no están involucrados, es decir no participa ni con su opinión, ni su involucramiento en la gestión de la oferta turística, por tanto, la propuesta cultural es estandarizada perdiendo las características genuinas de la cultura verídica.

\section{CONCLUSIONES}

Indudablemente toda intervención en un territorio que pretende generar el desarrollo traerá tanto impactos positivos como negativos en el 
curso de su proceso. Pero el ser humano como persona y miembro de una sociedad debe repensar sus acciones teniendo en cuenta como eje transversal la igualdad y equidad. Solo así puede lograr el bien común que como consecuencia traerá el bienestar anhelado. En el caso de las afro comunidades la estrategia del dúo turismo y cultura debe orientarse a fundar la diversidad identitaria, el protagonismo local, la memoria y cultura viva como bases para planificar, organizar y empoderar para la gestión del desarrollo turístico cultural como medio que promueva los fines supremos que se han propuesto los movimientos afrodescendientes. La reivindicación de sus aportes y derechos, la justicia para conformar parte de la sociedad y finalmente ser parte activa del desarrollo con sentido humanístico.

\section{REFERENCIAS BIBLIOGRÁFICAS}

Becerra, M. J. (2008). Estrategias de visibilización de la diáspora africana en América Latina y el Caribe durante el nuevo milenio. Ciencia Política, 3(5). Recuperado de https://revistas.unal.edu. co/index.php/cienciapol/article/view/17032

Benítez, N. \& Albuja, J. (2015). Legado de Personajes Afros y Afrodescendientes a la memoria social del Ecuador y el turismo cultural como estrategia de visibilización.

Benítez, N. \& Albuja, J. \& Tapia, G. (2015). Retrospectiva, visibilización y revalorización de la herencia cultural del pueblo Afro y Afroecuatoriano, a través del turismo comunitario, para el Buen Vivir. Revista Interamericana de Ambiente y Turismo - RIAT, 10(2), 166180. https://doi.org/10.4067/riatvol10iss2pp166-180\%0718-235X

Buitrago, A. (2006). Rodeados por las Murallas. Conflictos por el territorio en La Boquilla, Cartagena. Memorias. Revista Digital de Historia y Arqueología desde el Caribe, 3 (5), 0. Recuperado de https://www.redalyc.org/comocitar. oa?id=85530504

Camargo, B. \& Lawo-Sukam, A. (s. f.). San Basilio de Palenque (Re) visited: African Heritage, Tourism, and Development in Colombia, 23.

Campos Neves, S., \& Moquete Guzmán, S. (2008). Uma análise do novo movimento social afro em Ilhéus, BA, no contexto das "indústrias criativas" como modelo de desenvolvimento turístico local. Caderno Virtual de Turismo, 8 (2), 3445. Recuperado de https://www.redalyc.org/ articulo.oa?id=115415175004
Comisión Económica para América Latina y el Caribe. (2017). Situación de las personas afrodescendientes en América Latina y desafíos de políticas para la garantía de sus derechos. Naciones Unidas. Recuperado 4 de enero de 2019, de https://repositorio.cepal.org/bitstream/ handle/11362/42654/S1701063_es.pdf?sequence $=1 \&$ is Allowed $=\mathrm{y}$

Fuller, N. (2008). Turismo y cultura: entre el entusiasmo y el recelo. Fondo Editorial PUCP. Lima Perú. Recuperado 06 de diciembre del 2018, de http://blog.pucp.edu.pe/blog/wp-content/ uploads/sites/119/2010/11/01-Turismo-y-cultura-CS2.pdf

Guerrón, C. (2003). Repensando el desarrollo turístico, la globalización y la identidad: Perspectivas afro-antillanas sobre el turismo alternativo en Panamá. Pontificia Universidad Católica del Perú. Centro de Investigación en Geografía Aplicada.

Naciones Unidas. (2014) A/RES/69/16 - Programa de actividades del Decenio Internacional para los Afrodescendientes. Recuperado 6 de enero de 2019, de https://undocs.org/es/A/ RES/69/16

Naciones Unidas. (2009). Resolución adoptada por la Asamblea General de las Naciones Unidas. Revista Internacional del Trabajo, 128(1-2), 213-215. https://doi.org/10.1111/j.15649148.2009.00056.x

Organización de los Estados Americanos. (2016). Plan de acción del decenio de las y los afrodescendientes en las américas (2016-2025). AG/ RES. 2891 (XLVI-0/16). Recuperado 6 de enero de 2019, de https://www.oas.org/es/sadye/documentos/res-2891-16-es.pdf

Organización Mundial del Turismo. (1999). A/ RES/56/212 - Código Ético Mundial del Turismo. Recuperado 6 de enero de 2019, de http:// cf.cdn.unwto.org/sites/all/files/docpdf/gcetbrochureglobalcodees.pdf

Organización Mundial del Turismo. (s.f). Entender el turismo: Glosario Básico | Comunicación. (s. f.). Recuperado 6 de enero de 2019, de http:// media.unwto.org/es/content/entender-el-turismo-glosario-basico

Pazos Cárdenas, M. (2016). Industrias culturales «afropacíficas»: encrucijadas del multiculturalismo en la ciudad de Cali, Colombia. Antipoda. Revista de Antropología y Arqueología, (24), 75-90. https://doi.org/10.7440/antipoda24.2016.05

Pérez Amores, G. (2010). Orishas, turistas y practicantes. La comercialización del patrimonio religioso en Cuba: Un ejemplo de estrategia de 
revitalización identitaria y económica. PASOS. Revista de Turismo y Patrimonio Cultural, 8 (1), 167-184.

Tresserras, J. (2013). "El uso del patrimonio cultural para el turismo cultural: una mirada desde la comunidad para el desarrollo endógeno basado en un turismo sostenible y responsable". Ponencia. Seminario Internacional "El patrimo- nio cultural, un aporte al desarrollo endógeno" - Universidad Andina Simón Bolívar - UASB Quito, noviembre 2013.

UNESCO. (s. f.). Líneas Generales | Organización de las Naciones Unidas para la Educación, la Ciencia y la Cultura. Recuperado 6 de enero de 2019, de http://www.unesco.org/new/es/mexico/ work-areas/culture/ 